\title{
Magnitud del enfisema inducido por elastasa: Posible relación con el tipo de daño agudo pulmonar
}

\author{
ANDREA VECCHIOLA C.*, PILAR CELIS L.*, PABLO SANDOVAL R.*, ALFONSO LEÓN F.*, \\ ANDREA VILLAGRÁN T.*, ALEJANDRO RAMÍREZ M.* y GISELLA BORZONE T.*
}

\section{Elastase induced emphysema: Possible relationship with the type of acute lung injury}

Introduction: Intratracheal instillation of elastase induces diffuse alveolar damage and emphysema development. However, the Syrian Golden hamster develops more severe emphysema than the Sprague-Dawley rat. Although it is known that early events after elastase instillation determine the magnitude of emphysema development, it is not known if there are species differences in the initial pattern of lung response to elastase. Objective: To evaluate whether rats and hamster differ in the early lung response to elastase, using biochemical markers of acute lung injury. Results: Whereas the rat shows a large increase in alveolar-capillary permeability and few hemorrhagic changes, the hamster shows significant amount of hemorrhage and a small increase in alveolar capillary permeability. Conclusions: There are differences between rats and hamsters in the initial lung response to elastase that could influence the magnitude of emphysema development.

Key words: Acute lung injury, elastase, emphysema, animal models, hemorrhage.

\section{Resumen}

Introducción: El modelo de instilación intratraqueal de elastasa induce daño alveolar difuso y destrucción de la matriz extracelular con desarrollo de enfisema. Sin embargo, el hámster Syrian Golden desarrolla enfisema más severo que el de la rata Sprague-Dawley. Si bien se sabe que los eventos tempranos después de la instilación de elastasa determinan la magnitud del enfisema, se desconoce si existen diferencias entre especies en la respuesta pulmonar temprana. Objetivo: Evaluar si existen diferencias entre ratas y hamsters en la respuesta pulmonar inicial después de la elastasa, mediante el uso de marcadores bioquímicos de daño pulmonar agudo. Resultados: Mientras la rata experimenta un gran aumento de permeabilidad alvéolo-capilar y pocos fenómenos hemorrágicos, el hámster presenta abundante hemorragia y escaso aumento de la permeabilidad. Conclusiones: Existen diferencias entre ratas y hamsters en la respuesta pulmonar inicial frente a la elastasa, que podrían tener relación con las diferencias en magnitud del enfisema.

Palabras clave: Daño pulmonar agudo, elastasa, enfisema, modelos animales, hemorragia.

\section{Introducción}

Se acepta de manera general que, independientemente de la etiología, el pulmón reacciona de manera similar frente a distintos tipos de noxas ${ }^{1}$. La llegada de un agente injuriante a las estructuras alveolares induce un aumento de permeabilidad alvéolo-capilar, con la formación de edema intersticial y alveolar, grados variables de hemorragia y depósitos de fibrina. Esto va seguido del reclutamiento de células inflamatorias y la aparición de microatelectasias. Estas alteraciones morfológicas pueden adoptar el patrón de daño alveolar difuso (DAD) que compromete todas las estructuras del alvéolo (epitelio, endotelio y espacio intersticial) o el patrón de bronquiolitis obliterante con neumonía en organización (BOOP) que compromete la vía aérea distal

\footnotetext{
* Departamento de Enfermedades Respiratorias y Centro de Investigaciones Médicas, Pontificia Universidad Católica de Chile.
} 
y el parénquima peri-bronquiolar ${ }^{1-5}$. Estas alteraciones pueden regresar, con restitución ad integrum de la estructura alveolar o evolucionar al desarrollo de fibrosis y/o destrucción ${ }^{6}$. Se desconoce si variaciones morfológicas o bioquímicas en las etapas iniciales del daño pulmonar agudo pueden predecir un tipo de evolución u otro.

En un estudio previo de nuestro laboratorio, encontramos que 4 meses después de la instilación intratraqueal (IT) de una dosis similar de elastasa por kg de peso, el hámster Syrian Golden desarrolla un enfisema pulmonar severo con graves alteraciones de la mecánica respiratoria mientras que la rata Sprague Dawley desarrolla un enfisema leve, con menor repercusión funcio$\mathrm{nal}^{7,8}$. Si bien los factores capaces de influir en la desigualdad de respuesta frente a la elastasa instilada en las dos especies son múltiples, es posible que diferencias en el patrón de respuesta en las etapas iniciales del daño pulmonar frente a la elastasa guarden relación con la magnitud del enfisema resultante.

Con el objetivo de evaluar si entre estas dos especies existen diferencias en la respuesta inicial del pulmón frente a la instilación IT de elastasa, estudiamos algunos marcadores bioquímicos de daño pulmonar agudo en las primeras horas y días después de la instilación de la proteasa.

\section{Material y Método}

Modelo experimental de instilación intratraqueal de elastasa (ELA) ${ }^{8-10}$ : El estudio se realizó de acuerdo a las normas del Comité de Ética para el trabajo con animales de la Pontificia Universidad Católica de Chile. Ratas machos de la especie Sprague-Dawley, de $270 \pm 10 \mathrm{~g}$ de peso corporal y hamsters machos de la especie Syrian Golden, de $100 \pm 10 \mathrm{~g}$ de peso recibieron una dosis única de elastasa pancreática porcina (Sigma, 0,55 U/100 g de peso corporal diluidas en $0,5 \mathrm{ml}$ de $\mathrm{NaCl} 0,15 \mathrm{M}$ para las ratas y en $0,3 \mathrm{ml}$ para los hamsters) por vía IT. La solución de elastasa se preparó bajo condiciones estériles, inmediatamente antes de su administración IT con una aguja hipodérmica $25 \mathrm{G}$ bajo anestesia con Xilazina: Ketamina (10:75, mg/kg de peso). Para ello se realizó una pequeña incisión en la piel del cuello para separar los músculos cervicales y exponer la tráquea. Luego del cierre de la incisión y de la recuperación anestésica, los animales fueron mantenidos en el vivero, con un régimen de luz de 12 horas diarias y alimentados ad libitum con alimento estándar para roedores. Se estudiaron cuatro grupos de animales: 4 horas, 24 horas, 4 días y 7 días $(\mathrm{n}=$ 5 por grupo, descontando una letalidad del $20 \%$ ). El grupo control $(n=5)$ estuvo constituido por animales sin intervención, mantenidos en similares condiciones que los grupos experimentales $\mathrm{y}$ anestesiados al momento del sacrificio.

Inmediatamente después de la anestesia con Xilazina: Ketamina (10:75, mg/kg de peso), se realizó una laparotomía media para la extracción de 1-2 $\mathrm{ml}$ de sangre desde la vena cava inferior, para la separación del suero y el estudio de sus proteínas mediante electroforesis. Posteriormente los pulmones fueron resecados en bloque para la realización del procedimiento de lavado bronco-alveolar (LBA). Tres alícuotas de solución salina $0,15 \mathrm{M}$ de $3 \mathrm{ml}$ para hamsters y $9 \mathrm{ml}$ para rata, fueron instiladas, inmediatamente aspiradas, mezcladas y almacenadas a $-80{ }^{\circ} \mathrm{C}$ hasta su estudio.

Marcadores bioquímicos de daño pulmonar agudo: En el líquido obtenido por LBA se midió el contenido de proteínas totales por el método de Bradford $^{11}$, la concentración de hemoglobina utilizando la absorbancia a $414 \mathrm{~nm}$ y se realizó electroforesis en gel de poliacrilamida para la separación de las proteínas, las cuales fueron teñidas con azul de Coomassie (Coomassie R250 en 10\%-ácido acético/45\%-metanol).

En un grupo especial de animales $(n=20$ para cada especie) se resecó, bajo anestesia, el pulmón izquierdo para ser inmediatamente pesado (peso húmedo) y dejado en una estufa a $30^{\circ} \mathrm{C}$ hasta obtener estabilidad en su peso (peso seco) para el cálculo de la razón peso seco/peso húmedo a distintos tiempos después de la instilación de elastasa (control, 4 h, 24 h y 4 días).

Análisis estadístico: Se utilizó análisis de varianza (ANOVA) y la prueba post-hoc de Tukey $^{12}$. Un valor de $p<$ de 0,05 fue considerado estadísticamente significativo.

\section{Resultados}

\section{Marcadores de aumento de la permeabilidad alvéolo-capilar a distintos tiempos después de la instilación IT de elastasa.}

a) proteinas totales en el lavado broncoalveolar

La Figura 1 muestra en el panel superior, geles representativos teñidos con azul de Coomassie con el perfil electroforético de las proteínas presentes en el lavado broncoalveolar 


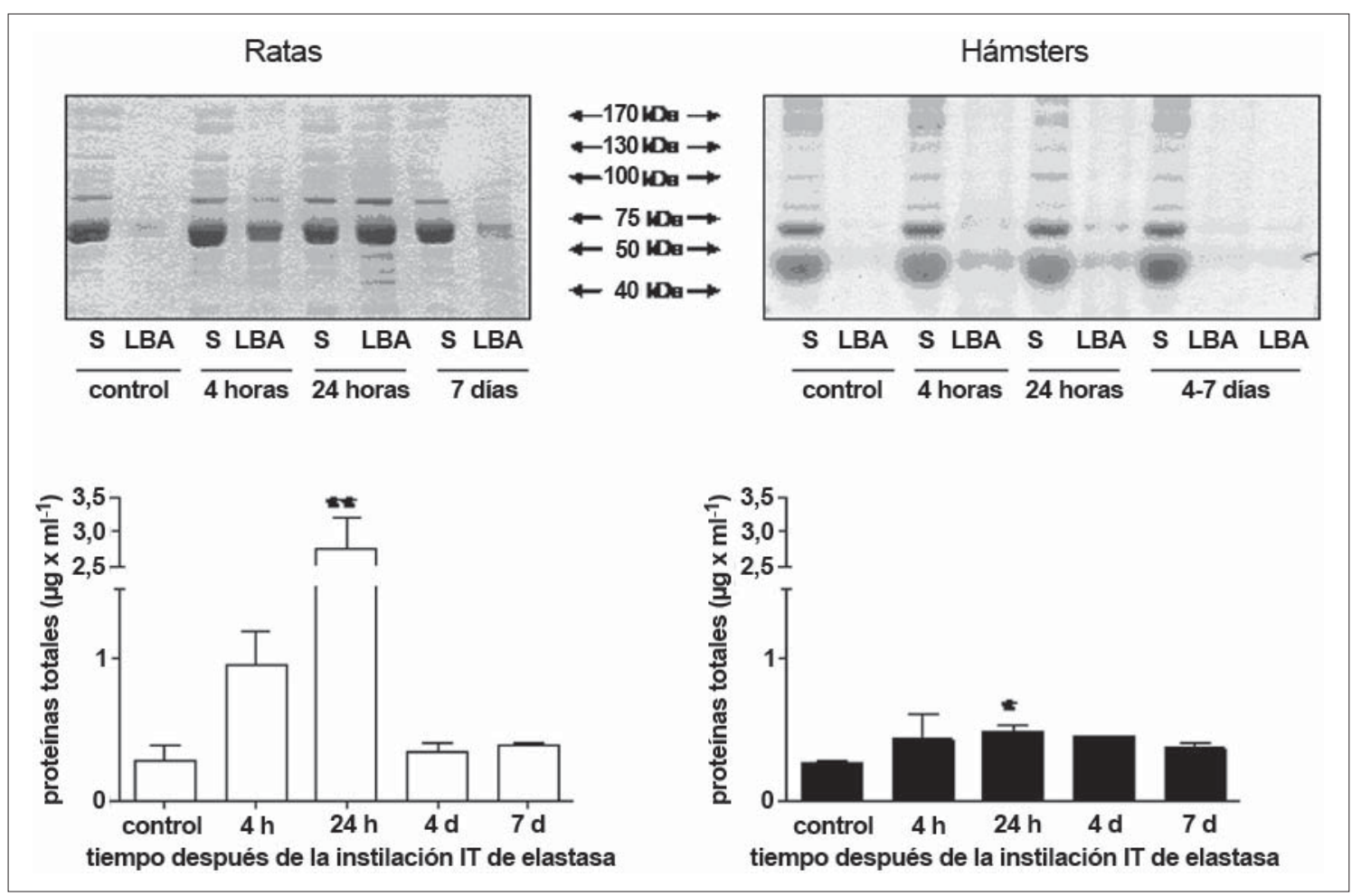

Figura 1. Curso temporal de los cambios en el perfil electroforético y la concentración de las proteínas totales en el lavado broncoalveolar (LBA) después de la instilación intratraqueal de elastasa. En el panel superior se muestra 1 gel representativo para cada especie, teñido con azul de Coomassie, para una muestra de suero (S) y (LBA) proveniente de un mismo animal en condiciones basales y a distintos tiempos después de la instilación de elastasa. En la rata, las proteínas del LBA aumentan progresivamente hasta tener un perfil similar al del suero a las $24 \mathrm{~h}$ con retorno al perfil del LBA control a los 7 días. En el hámster si bien hay un aumento en las proteínas del LBA, el perfil no llega a asemejarse al del suero ya que predominan proteínas de menor tamaño molecular que en la rata. En el panel inferior, las barras representan promedio \pm error estándar de la concentración de proteínas totales en el LBA de ratas (barras blancas) y hamsters (barras negras) medidas con el método de Bradford, a distintos tiempos después de la instilación intratraqueal (IT) de elastasa. $* * p<0,01 * \mathrm{p}<0,05$ en relación al control; $\mathrm{d}=$ días; $\mathrm{h}=$ horas.

(LBA) y el suero (S) de ratas y hamsters a distintos tiempos después de la instilación de elastasa. Las muestras de suero fueron tomadas del mismo animal del cual se obtuvo el LBA. Es posible observar para ambas especies, que en el grupo control las muestras de LBA y de suero difieren en el contenido y movilidad de las proteínas, encontrándose muy pocas proteínas en el lavado y una gran variedad de éstas en el suero.

En la rata, a partir de las cuatro horas después de la instilación IT de elastasa, el LBA presenta un cambio en el perfil de las proteínas, apareciendo proteínas de distinto peso molecular que están presentes en el suero. Ese fenómeno se acentúa a las 24 horas, en que el LBA adquiere un perfil de proteínas muy similar al del suero, como reflejo de un gran aumento de la permeabilidad alvéolo-capilar.
En el hámster, si bien es posible observar la aparición de algunas proteínas séricas en el LBA después de la instilación de elastasa, el perfil electroforético de éstas no llega a parecerse al perfil de las proteínas del suero.

Por otra parte, a los siete días, el perfil de las proteínas del LBA se hace similar al del grupo control en ambas especies.

El panel inferior de la Figura 1 ilustra las variaciones en la concentración de proteínas totales en el LBA medida con el método de Bradford a los distintos tiempos después de la instilación IT de elastasa para ambas especies. Mientras en la rata la concentración de proteínas aumenta 3,4 veces a las 4 horas $(\mathrm{p}<0,05)$ y 9,6 veces a las 24 horas $(p<0,01)$, en el hámster el aumento en la concentración de proteínas es sólo de dos veces con respecto al nivel basal $(\mathrm{p}<0,05)$. 


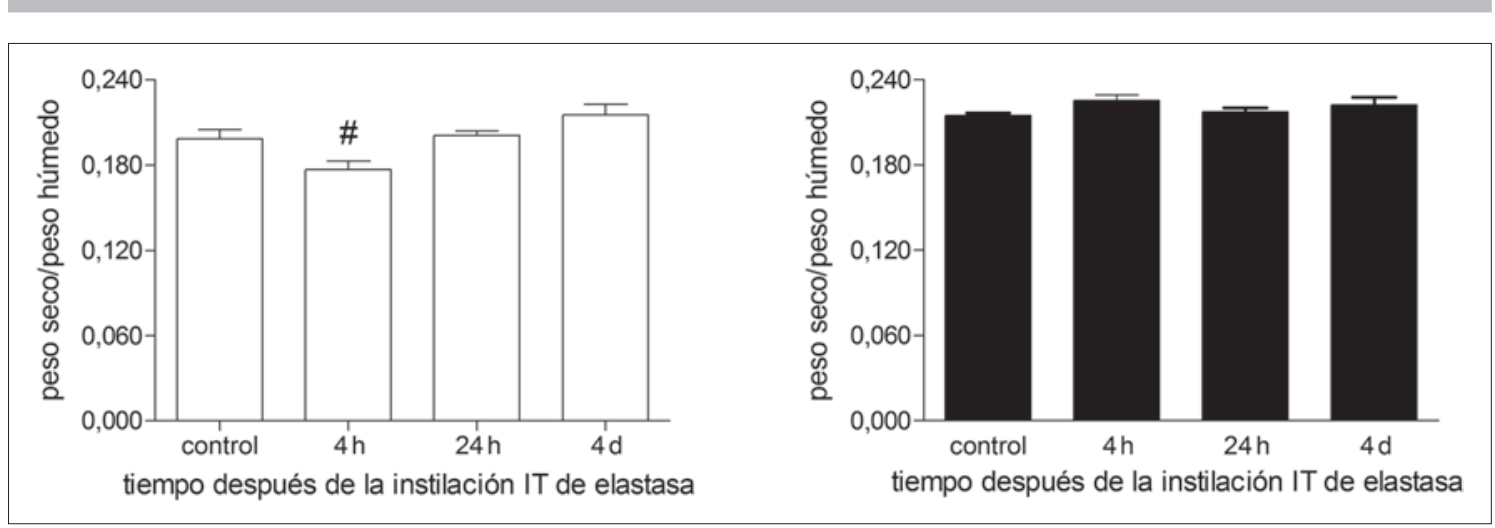

Figura 2. Relación peso seco/peso húmedo del pulmón a distintos tiempos después de la instilación intratraqueal (IT) de elastasa para ambas especies. Las barras corresponden al promedio \pm error estándar. Ratas (barras blancas) y hámsters (barras negras). $\# \mathrm{p}<0,05$ en relación a los 4 días; $\mathrm{d}=$ días; $\mathrm{h}=$ horas.

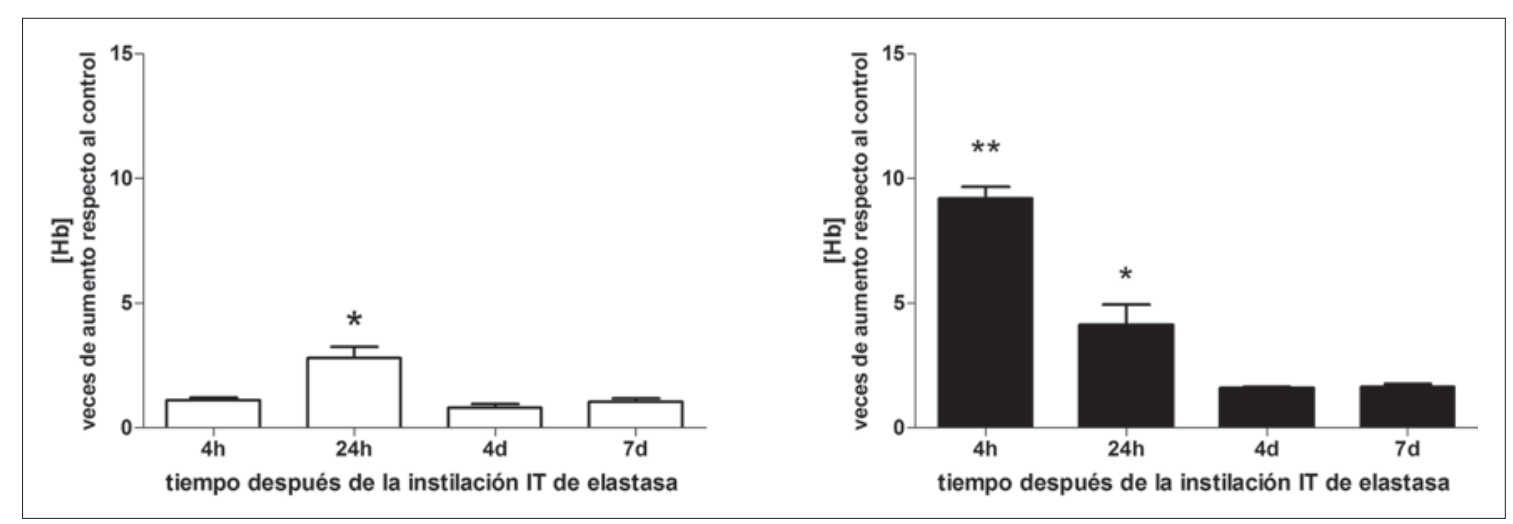

Figura 3. Cambios en la concentración de hemoglobina $[\mathrm{Hb}]$ en el LBA a distintos tiempos después de la instilación intratraqueal (IT) de elastasa, expresada como veces de aumento en relación al control. Las barras corresponden al promedio \pm error estándar de los resultados obtenidos en 5 experimentos independientes. Ratas (barras blancas) y hámsters (barras negras). ${ }^{* *} \mathrm{p}<0,01 * \mathrm{p}<0,05$ en relación al control; $\mathrm{d}=\mathrm{días} ; \mathrm{h}=$ horas.

\section{b) Razón peso seco/peso húmedo del pulmón}

La Figura 2 muestra los cambios en la razón peso seco/peso húmedo del pulmón de ratas y hámsters a distintos tiempos después de la instilación de elastasa. En la rata, esta razón muestra una significativa disminución a las 4 horas después de la instilación de la elastasa $(\mathrm{p}<0,05)$ con normalización a las $24 \mathrm{~h}$. En el hámster en cambio, no encontramos cambio significativo de esta razón.

\section{Hemorragia pulmonar en el lavado broncoalveolar}

La Figura 3 ilustra los cambios en la concentración de hemoglobina en el LBA después de la instilación de elastasa en la rata y el hámster. En la rata se observa un aumento en la concentración de hemoglobina entre las 4 horas y los 4 días, con diferencia significativa sólo a las 24 horas $(\mathrm{p}<0,05)$ cuando la concentración de hemoglobina alcanza 2,8 veces el valor del grupo control.

En el hámster en cambio, encontramos que el mayor aumento en la concentración de hemoglobina se produce a las cuatro horas después de la instilación de la elastasa. Dicho aumento es de 7,5 veces el valor del grupo control $(p<0,01)$. La concentración de hemoglobina se mantiene alta a las $24 \mathrm{~h}$ con 5,7 veces el valor del grupo control $(\mathrm{p}<0,05)$ y retorna a valores basales a los 4 días.

\section{Discusión}

Los resultados de este estudio muestran que la rata Sprague-Dawley y el hámster Syrian Golden difieren en el comportamiento de los 
marcadores bioquímicos utilizados para evaluar el daño pulmonar agudo después de la instilación IT de elastasa. En el hámster, el patrón de respuesta pulmonar inicial se caracteriza por una significativa participación de fenómenos hemorrágicos y escasa evidencia de aumento de la permeabilidad alvéolo-capilar. En la rata en cambio, los fenómenos hemorrágicos, si bien están presentes, son de menor magnitud y más tardíos. En esta especie predominan las evidencias que apoyan la existencia de un significativo aumento de la permeabilidad alvéolo-capilar.

Se ha descrito que horas después de la instilación IT de elastasa en los pulmones de la rata y el hámster es posible encontrar edema, hemorragia y acumulación de células inflamatorias, alteraciones morfológicas que al desaparecer, dejan en evidencia la formación de grandes espacios aéreos ${ }^{1,10}$. Sin embargo, en la literatura se extrapolan resultados de una especie a la otra y no se han hecho intentos por estudiar si existen diferencias entre estas especies, en el patrón de respuesta pulmonar inicial frente a la instilación de la proteasa. Por otra parte, Lucey y $\operatorname{cols}^{10}$, han postulado que la magnitud del daño enfisematoso final secundario a la instilación IT de elastasa se determina dentro de las primeras horas después de la instilación de la proteasa. Por lo anterior, resulta relevante estudiar el patrón de respuesta pulmonar en las primeras horas y días después de la instilación IT de elastasa, con el objetivo de identificar factores pronósticos de mayor daño pulmonar frente a la elastasa.

El aumento de la permeabilidad alvéolo-capilar, ha sido considerado tradicionalmente como el principal marcador de daño pulmonar agudo, ya que existe correlación entre la magnitud del aumento de permeabilidad y el grado de alteración del intercambio gaseoso y la gravedad del cuadro clínico ${ }^{13}$. Sin embargo, nuestros resultados muestran que la especie con mayor evidencia de aumento de la permeabilidad alvéolo-capilar desarrolla menor daño crónico, mientras que la especie con menor alteración de la permeabilidad desarrolla enfisema más severo.

Resulta evidente que el estudio de los marcadores de aumento de permeabilidad alvéolo-capilar de manera aislada y sin considerar otros marcadores de daño agudo, no es suficiente para evaluar la relación entre el patrón de respuesta pulmonar inicial frente a la elastasa y la magnitud del daño enfisematoso resultante.

Una posible explicación para esto podría estar en el hecho que el aumento de la permeabilidad como parte del proceso inflamatorio frente a una noxa, constituye también una respuesta defensiva del organismo. El aumento de la permeabilidad alvéolo-capilar favorece el paso de proteínas séricas al intersticio pulmonar y a los espacios aéreos, entre los cuales están las anti-

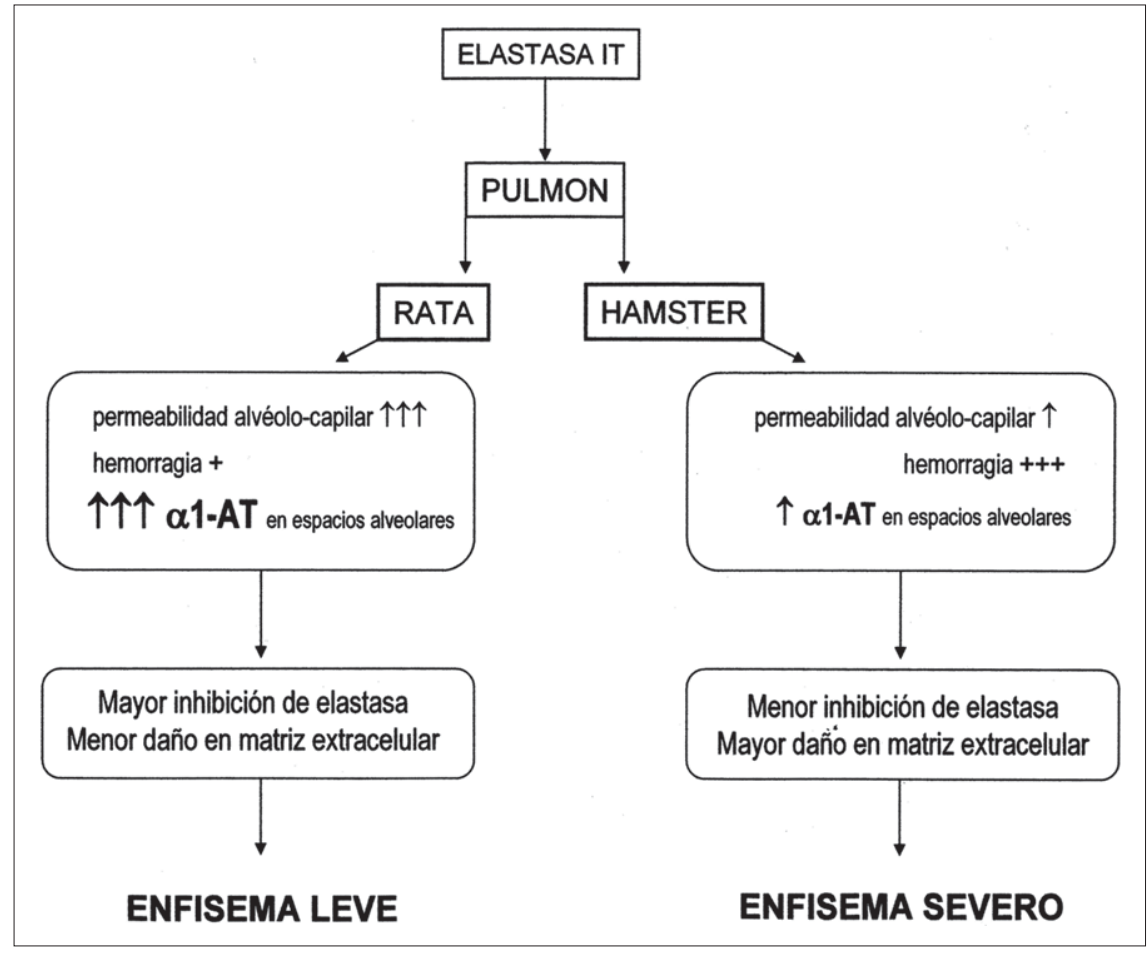

Figura 4. Hipótesis de posible relación entre el patrón de respuesta pulmonar inicial después de la instilación de elastasa y el enfisema resultante. 
proteasas, como por ejemplo la alfa 1-antitripsina, que defienden al pulmón del daño por proteasas ${ }^{14}$. Si se considera que la rata posee una mayor concentración sérica de alfa 1-antitrip$\operatorname{sina}^{8} \mathrm{y}$ al mismo tiempo experimenta un mayor aumento de la permeabilidad alvéolo-capilar, que favorece la llegada de esta antiproteasa específica para la elastasa instilada, es posible que en esta especie, la elastasa tenga menos facilidad para interactuar con los componentes de la matriz extracelular, que en el hámster, determinando menos daño enfisematoso crónico.

Nuestros resultados muestran que en el hámster, que desarrolla enfisema severo, los tres marcadores bioquímicos utilizados para evaluar el aumento de la permeabilidad alvéolo-capilar experimentan pocos cambios en comparación con la rata. Si la hipótesis de que el aumento de permeabilidad tiene un papel protector es correcta, entonces en el hámster, la llegada de antiproteasas desde el suero hacia los espacios aéreos sería menor que en la rata, al tener un menor aumento de permeabilidad y al mismo tiempo, una menor concentración sérica de alfa1antitripsina ${ }^{8}$, favoreciéndose la interacción de la elastasa con los componentes de la matriz, mayor magnitud de fenómenos hemorrágicos y eventualmente mayor daño enfisematoso (Figura 4).

En conclusión, existen diferencias en la respuesta pulmonar inicial de la rata Sprague-Dawley y la del hámster Syrian Golden después de la instilación IT de elastasa, sin embargo, la relación entre estas diferencias y la distinta magnitud del enfisema que dichas especies desarrollan con el tiempo es por ahora especulativa. Proponemos que para evaluar de mejor manera el daño alveolar difuso inducido por la elastasa y otras noxas se consideren distintos marcadores bioquímicos de daño alvéolo-capilar.

\section{Bibliografía}

1.- KATZENSTEIN A. Acute lung injury patterns: Diffuse alveolar damage and bronchiolitis obliterans-organizing pneumonia. En: Katzenstein and Askin's Surgical Pathology of Non-Neoplastic Lung Disease. Philadelphia. WB Saunders Company 1997; 14-47.

2.- ANDERSON W, STRICKLAND M, TSAI S, HAGLIN J. Light microscopic and ultrastructural study of adverse effects of oxygen therapy on the neonate lung. Am J Pathol 1973; 73: 327-48.

3.- CEDERBERG A, HELLSTEN S, MIORNER G. Oxygen treatment and hyaline pulmonary membranes in adults. Acta Pathol Microbiol Scand 1965; 64: 450-58.
4.- HOMMA S, JONES R, QVIST J, ZAPOL W M, REID L. Pulmonary vascular lesions in the adult respiratory distress syndrome caused by inhalation of zinc chloride smoke: A morphometric study. Hum Pathol 1992; 23 : 45-50.

5.- TAKAHASHI T, TAKAHASHI Y, NIO M. Remodeling of the alveolar structure in the paraquat lung of humans: A morphometric study. Hum Pathol 1994; 25: 702-8.

6.- MCELVANEY N, CRYSTAL R. Proteases and lung Injury. En: R. Crystal, J West y cols. The Lung Scientific Foundations. Philadelphia. Lippincot-Raven Publisher 1997; 2205-18.

7.- BORZONE G, LIBERONA L, OLMOS P, SÁEZ C, MENESES M, REYES T, et al. Rat and hamster species differences in susceptibility to elastase-induced pulmonary emphysema relate to differences in elastase inhibitory capacity. Am J Physiol Regul Integr Comp Physiol 2007; 293: R1342-49.

8.- BORZONE G, RAMÍREZ B, REYES T, MORENO R, LISBOA C, GONZÁLEZ S. Experimental pulmonary emphysema in the rat. Inflammatory phenomena and progression of lung damage. Rev Méd Chile 1998; 126: $1153-60$.

9.- SNIDER G L, SHERTER C B. A one-year study of the evolution of elastase-induced emphysema in hamsters. J Appl Physiol 1977; 43: 721-29.

10.- LUCEY E C, GOLDSTEIN R H, STONE P J, SNIDER G L. Remodeling of alveolar walls after elastase treatment of hamsters. Results of elastin and collagen mRNA in situ hybridization. Am J Respir Crit Care Med 1998; 158: 555-64.

11.- BRADFORD M M. A rapid and sensitive method for quantitation of microgram quantities of protein utilizing the principle of protein dye binding. Anal Biochem 1976; 72: 248-54.

12.- TUKEY J W. Some selected quick and easy methods of statistical analysis. Trans NY Acad Sci 1953; 16: 88-97.

13.- WEILAND J E, DAVIS W B, HOLTER J F, MOHAMMED J R, DORINSKI P M, GADEK J E. Lung neutrophils in the adult respiratory distress syndrome clinical and pathophysiologic significance. Am Rev Respir Dis. 1986; 133: 218-25.

14.- MCELVANEY N, CRYSTAL R. Antiproteases and lung defense. En: R. Crystal, J West y cols. The Lung Scientific Foundations. Philadelphia. Lippincot-Raven Publisher 1997; 2219-35.

\section{Correspondencia a:}

Dra. Andrea Vecchiola Cárdenas

Departamento de Enfermedades Respiratorias y Centro de Investigaciones Médicas. Facultad de Medicina.

Pontificia Universidad Católica de Chile.

Marcoleta 391, Piso 3.

Teléfono: 354-3815; Fax: 633-5255

E-mail: avecchi@med.puc.cl 